\title{
New Liquid Crystalline Polyurethane Elastomers Containing Thiazolo [5,4d] Thiazole Moiety: Synthesis and Properties
}

\author{
Issam Ahmed Mohammed and Mohamed Rashidah Hamidi
}

Additional information is available at the end of the chapter

http://dx.doi.org/10.5772/36784

\section{Introduction}

Originally there are three states of matter; solid, liquid and gas. The emergence of an exotic and extraordinary form of matter, which is known as liquid crystal has been considered as one of the major breakthrough in polymer science. Liquid crystal can be defined as an intermediate of solid (crystal) and liquid (Knight \& Vollrath, 2002) where the molecules have the capabilities to flow like a liquid (mobility) as well as possessing the common property associated to solid, which is the degree of order (Doldeny \& Alder, 1998). In addition, liquid crystal materials are self assembling by nature and can offer a very elegant and effective way of controlling and tuning the physical properties that ultimately define the self-organizing and self assembly process (Zhang et.al., 2008). One of the exciting developments involving this unique material is the introduction of liquid crystalline behavior in polyurethane elastomers (PUE) where the first of this kind was synthesized by Iimura in 1981 (Lin et.al., 2001).

Polyurethane $[\mathrm{PU}]$ is one of the most versatile class of polymeric materials known today. Wide variety of structural changes can be produced with the different hydroxyl compounds and isocyanates leading to a wide spectrum of properties and applications (Yeganeh et.al., 2007). It contains a high concentration of polar groups, in particular the urethane group, resulting from isocyanate-hydroxyl reactions. The interactions between these polar entities are of great importance in determining the properties of PU of all types (Lee et.al., 1999) besides the composition and characteristic of the polyol, diisocyanates and the additives utilized during the synthesis (Pachecho et.al., 2009).

High toughness, excellent wear and tear properties and good oil resistance are among the advantages displayed by PUE (Wright \& Cumming, 1969). Moreover, not only they have good mechanical and physical properties, PUE are also benefited with biocompatibility 
characteristics for biomedical applications (Barikani et.al., 2009). Despite all the great aforementioned properties, modifications and improvements are done to conventional PUE in order to meet the qualities in more advanced applications.

Diisocyanates, polyol and low molecular weight diamine or diol (chain extender) are the basic building blocks of conventional PUE (Yeganeh \& Mehdizadeh, 2009). In order to synthesize liquid crystal polyurethane elastomers (LCPUE), the low molecular weight diamine or diol used in conventional PUE was substituted with the mesogenic unit. Incorporation of geometrically anisotropic moieties (mesogenic unit) within polymer architecture can drive the formation of liquid crystalline phase from strictly steric repulsion considerations (Abe \& Ballauf, 1991; Rowan \& Mather, 2007). Furthermore, the insertion of mesogenic unit in the backbone of PUE will impart unique physical properties to the polymer and also improve its mechanical, optical and electrical characteristics (Jia et.al., 1996).

Various mesomorphic behaviors are exhibited with different types of mesogenic units in preparation of LCPUE. In this research work, mesogens consumed were thiazolo [5.4d] thiazoles based and it is known as an important class of biycyclic aromatic molecule comprising two fused thiazole rings (Knighton et.al., 2010). Thiazolothiazole rigid fused ring can enhance the rigidity of the polymer and the conjugation (Osaka et.al, 2007) which makes it a best candidate to be part of the hard segment in the LCPUE network. The hard segments consisted of either 2,5-bis(4-hydroxyphenyl) thiazolo-[5,4d] thiazole or 2,5-bis(4-hydroxy-3methoxyphenyl) thiazolo [5,4d] thiazole and 4,4'- methylene diphenyl diisocyanate (MDI). As for the soft segments, polyethylene glycol (PEG) 1000, 2000 and 3000 were involved.

The ultimate aim of this work is to synthesize new LCPUE with the presence of thiazolo[5,4d] thiazole as a chain extender. Study and analysis were carried out to determine the effects and consequences of the introduction of thiazolo-[5,4d] thiazole moiety and the influence of various lengths of polyols on the properties of LCPUE.

\section{Experimental}

\subsection{Materials}

Vanillin and 4, 4'-methylene diphenyl diisocyanate (MDI) were purchased from Aldrich Co. (United States). Rubeanic Acid (dithiooxiamide) and 4-hydroxybenzaldehyde were obtained from MERCK Co. (Germany). Polyethylene glycol with molecular weight of 3000, 2000 and 1000 (PEG: $\mathrm{Mn}=3000,2000$ and 1000) were purchased from Fluka Chemica (Switzerland). All the chemicals were utilized as received without any further purification. N,NDimethylformamide purchased from Aldrich (United States) was distilled over Calcium Hydride $\left(\mathrm{CaH}_{2}\right)$ through vacuum distillation before being used.

\subsection{Synthesis of monomers and polymers}

\subsubsection{Synthesis of 2,5-bis(4-hydroxyphenyl)thiazolo-[5,4d] thiazole (I)}

Briefly, $3 \mathrm{~g}$ (25 mmol) of dithiooxamide (Rubeanic acid) and $15 \mathrm{~g}$ (123 mmol) of 4hydroxybenzaldehyde with the presence of $9 \mathrm{~g}(97 \mathrm{mmol})$ of phenol were charged all at once 
in a $500 \mathrm{ml}$ round bottom flask fitted with condenser and left to be refluxed for $2 \mathrm{~h}$. Precipitates were obtained by pouring the hot mixtures to the cold water. Subsequently, the yield was filtered off and washed with ethanol followed by ether. The product obtained was dried at $70^{\circ} \mathrm{C}$ in a vacuum oven for 24 hours. Recrystallization from cyclohexanone was performed giving an orange-yellowish powder. Yield: $35 \%$ with melting point $364^{\circ} \mathrm{C}$. Fourier transform infrared (FTIR; KBr, cm-1): $3492(-\mathrm{OH}), 1606(\mathrm{C}=\mathrm{N}), 1596$ (C=C), 855 (p-substituted benzene). ${ }^{1} \mathrm{H}$ NMR (400 MHz, DMSO-d 6 ppm): $\delta_{\mathrm{H}} 7.12$ (m, aromatic protons), 9.8 (s, -OH). Elemental analysis: Found: $\mathrm{C}, 59.16 ; \mathrm{H}, 3.28 ; \mathrm{N}, 8.84, \mathrm{C}_{16} \mathrm{H}_{10} \mathrm{~N}_{2} \mathrm{O}_{2} \mathrm{~S}_{2}$ Calc.: C, 58.89; H, 3.09, N, 8.59.

\subsubsection{Synthesis of 2,5-bis(4-hydroxy-3-methoxyphenyl) thiazolo [5,4d] thiazole (II)}

The same procedure was applied to the synthesis of 2,5-bis(4-hydroxy-3-methoxyphenyl) thiazolo $[5,4 \mathrm{~d}]$ thiazole except that 4-hydroxybenzaldehyde was substituted with vanillin. Orange-yellowish powder was obtained as the end product. Yield: $26 \%$ with the melting point of $259^{\circ} \mathrm{C}$. Fourier transform infrared (FT-IR; KBr disc): $3534 \mathrm{~cm}^{-1}(\mathrm{OH}), 1608 \mathrm{~cm}^{-1}(\mathrm{C}=\mathrm{N})$, $1510 \mathrm{~cm}^{-1}(\mathrm{C}=\mathrm{C}), 842 \mathrm{~cm}^{-1}$ (-CH out of plane). ${ }^{1} \mathrm{H}-\mathrm{NMR}$ (400 MHz, DMSO-d 6 ppm): $\delta$ н 7.09 (m, aromatic protons), $9.5(\mathrm{~s},-\mathrm{OH})$ and $3.87\left(\mathrm{~s}, \mathrm{OCH}_{3}\right)$. Elemental analysis: Found: $\mathrm{C}, 55.60 ; \mathrm{H}$, $4.03 ; \mathrm{N}, 6.89, \mathrm{C}_{18} \mathrm{H}_{14} \mathrm{~N}_{2} \mathrm{O}_{4} \mathrm{~S}_{2}$ Calc.: $\mathrm{C}, 55.95 ; \mathrm{H}, 3.62, \mathrm{~N}, 7.25$.

\subsubsection{Synthesis of liquid crystalline polyurethane elastomers (LCPUE)}

Preparation of LCPUE was achieved by two steps solution polymerization reaction, where isocyanate terminated pre-polymer was synthesized initially in the first stage. To produce pre-polymer, $0.01 \mathrm{~mol}$ of PEG (Mw $=1000,2000$, and 3000) and $0.02 \mathrm{~mol}$ of MDI were mixed in $500 \mathrm{ml}$ of reactor flask equipped with condenser, thermometer, nitrogen inlet and mechanical stirrer. The mixture was allowed to be stirred and heated for $4 \mathrm{~h}$ at $70^{\circ} \mathrm{C}$ in the presence of $15 \mathrm{ml}$ of DMF as solvent and nitrogen gas was kept flowing to provide inert atmosphere. The reaction was followed by chain extension process, using either compound (I) or (II), where the chain extender was added dropwise within $1 \mathrm{~h}$ to complete the formation of LCPUE. Subsequently, the temperature was increased to $100^{\circ} \mathrm{C}$ and the reaction continued for another $9 \mathrm{hr}$. The hot viscous solution was then poured into $200 \mathrm{ml}$ of cold water for precipitation, before subjected to filtration. Later, the filtered product was washed with ethanol several times and finally with ether, before being dried overnight in a vacuum oven at $60^{\circ} \mathrm{C}$.

\subsection{Measurements}

100mg mixture of samples and $\mathrm{KBr}$ (grounded) were pressed into translucent disc before being subjected to Nicolar Avatar Model 360 Fourier Transform infrared spectrometer devices to obtain FT-IR spectra. Data was collected in the range of $4000-400 \mathrm{~cm}^{-1} .{ }^{1} \mathrm{H}-\mathrm{NMR}$ and ${ }^{13} \mathrm{C}$-NMR spectra were obtained using Bruker $400 \mathrm{MHz}$ NMR spectrometer consuming DMSO- $\mathrm{d}_{6}$ as solvent and TMS as internal standard. Thermal stability of LCPUE was determined by thermogravimetric analyzer (Perkin Elmer Pyris series 6) under nitrogen purge and with $10^{\circ} \mathrm{C} / \mathrm{min}$ of heating rate and the heating was done up to $800^{\circ} \mathrm{C}$. Liquid crystalline behavior was verified by means of differential scanning calorimetry (DSC) to 
observe the behavior of polymers such as glass transition point $\left(\mathrm{T}_{\mathrm{g}}\right)$, melting point $\left(\mathrm{T}_{\mathrm{m}}\right)$ and isotropic temperature $\left(\mathrm{T}_{\mathrm{i}}\right)$. It was conducted utilizing Perkin Elmer Pyris Series 7 thermal analyzer under Nitrogen flux at $10^{\circ} \mathrm{C} / \mathrm{min}$ rate of heating. Textures of mesomorphic phases were displayed by Nikon Eclipse E600 polarized microscope equipped with MS600 Linkam Hot stage and SONY CCD-IRIS Color Video Camera. The heating rate was $5^{\circ} \mathrm{C} / \mathrm{min}$ and $10^{\circ} \mathrm{C} / \mathrm{min}$ for the cooling rate. Sample was placed between two thin round glasses and it was then transferred onto microscope fitted with the hot stage to be analyzed. Siemens X-ray Diffractometer model D5000 equipped with a CuK $\alpha$ target at $40 \mathrm{KV}$ and $40 \mathrm{~mA}$ was used in obtaining X-ray scattering curve. Tensile strain properties of LCPUE films were measured by Instron Testing instrument at a constant speed of $500 \mathrm{~mm} / \mathrm{min}$ (speed) where the measurements were performed at room temperature. Brookfield viscometer was used to measure the fluid viscosity where suitable spindle and speed were chosen and it was also performed at room temperature.

\section{Results and discussion}

\subsection{Preparation of chain extender}

The preparation of 2,5-bis(4-hydroxyphenyl)thiazolo-[5,4d] thiazole and 2,5-bis(4-hydroxy3-methoxyphenyl) thiazolo [5,4d] thiazole were conducted according to the reaction shown in Scheme 1. The starting reagent involved for the synthesis of both the compounds were rubeanic acid and either 4-hydroxybenzaldehyde or vanillin with the presence of phenol. Subsequently, both chain extenders prepared were being used in the preparation of LCPUE. Identification of the chemical structures of the aforementioned products was monitored primarily with FT-IR spectroscopy and further confirmation was carried out by ${ }^{1} \mathrm{H}-\mathrm{NMR}$ spectrophotometer.

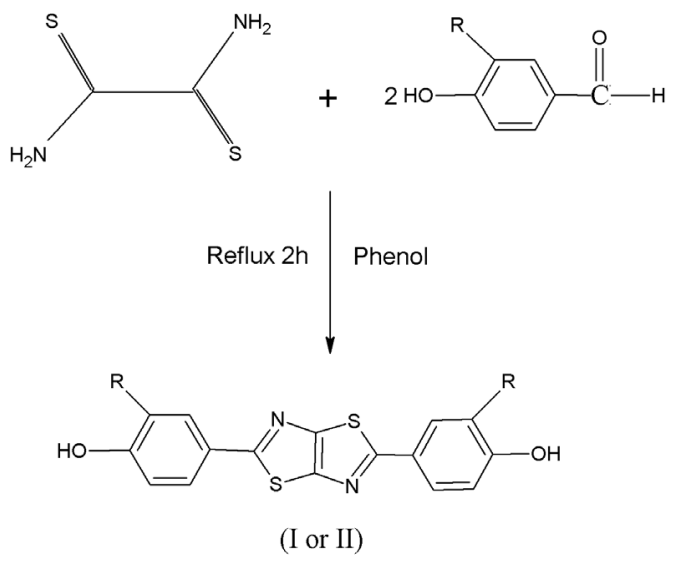

where $\mathrm{R}=-\mathrm{H}(\mathrm{I})$ or $-\mathrm{OCH}_{3}$ (II)

Scheme 1. Preparation of Compound I and II 


\subsection{Polymer synthesis}

LCPUE based on thiazolo [5,4d] thiazoles moiety were synthesized from long chain of diol (PEG 3000, 2000 and 1000) with an excess of diisocyanate (MDI) via addition reaction to give the terminal reactive group which results in the formation of 'extended diisocyanate' or isocyanate pre-polymer. Then, 2,5-bis(4-hydroxyphenyl) thiazolo-[5,4d] thiazole [I] and 2,5bis(4-hydroxy-3- methoxyphenyl) thiazolo [5,4d] thiazole [II] were added acting as a chain extender in order to convert the pre-polymer into long chain LCPUE. The general route for the preparation of LCPUE was outlined in Scheme 2, yield and viscosity of LCPUE were listed in Table 1 and the data showed that the range of the viscosities and yields obtained were 10,744 to $40692 \mathrm{cP}$ and 76-87\%, respectively. Range of the viscosities obtained also provides the information of the molecular weight of each polymer synthesized where high value of viscosity indicates high molecular weight of the polymer produced and vice versa (Bagheri \& Pourmoazzen, 2008). In this case, all LCPUE samples displayed fairly high molecular weight in accordance with the results demonstrated.

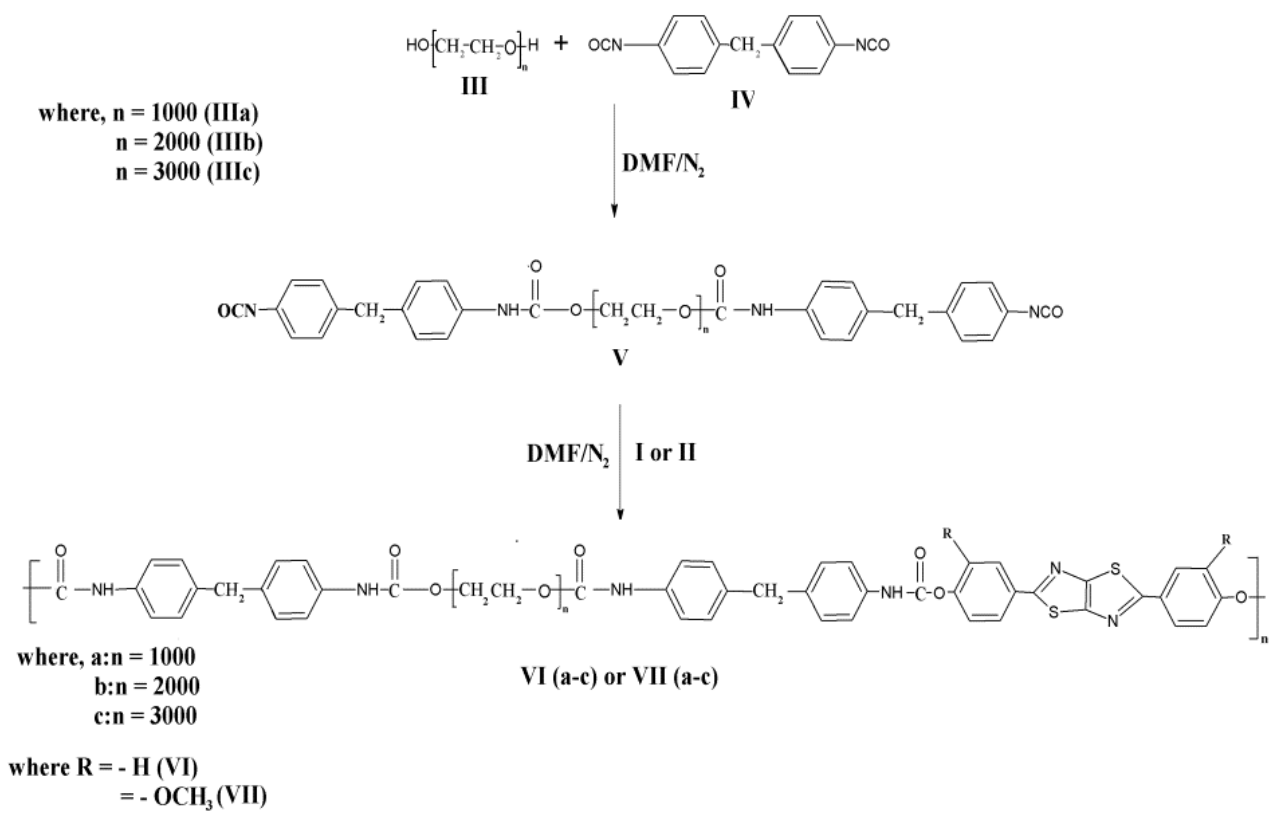

Scheme 2. General route for the preparation of LCPUE VI (a-c) and VII (a-c)

\subsection{Structural elucidation}

FT-IR was employed to verify functional groups of the pre-polymer, compound I and II, and LCPUE. Prior to the formation of LCPUE which is referring to the pre-polymer state, in the region of $2270 \mathrm{~cm}^{-1}$ a peak was observed which was assigned to $-\mathrm{N}=\mathrm{C}=\mathrm{O}$ - 
(diisocyanate) whereas according to the IR spectra of compound I and II, a peak was found at $3492 \mathrm{~cm}^{-1}$ and $3334 \mathrm{~cm}^{-1}$ which corresponds to $-\mathrm{OH}$ functional group in the chemical structure. The disappearance of both the bands of $-\mathrm{N}=\mathrm{C}=\mathrm{O}$ - in pre-polymer and $\mathrm{OH}$ of compound I and II, indicates the completion of the reaction of preparation of LCPUE and this fact was also supported with the appearance of new absorption bands at $3356.84 \mathrm{~cm}^{-1}$ ( N-H- stretching ) and $1782.5 \mathrm{~cm}^{-1}$ (carbonyl group) which were attributed to the urethane linkage, -NHCOO- (Zhang et al., 2008; Issam, 2007). Furthermore, the peak at $2884.89 \mathrm{~cm}^{-1}$ was ascribed to $-\mathrm{CH}$ stretching, whereas the band representing $\mathrm{C}=\mathrm{C}$ aromatic can be found at $1598.59 \mathrm{~cm}^{-1}$. Figure 1 displayed the FTI-R spectrum of LCPUE VIIa and based on the results obtained, the characteristic absorption bands of FT-IR spectra for the other LCPUE were almost identical to one another. The fact that differentiates LCPUE VI and LCPUE VII was the presence of the methoxy group and it was proven in the FTIR spectrum of LCPUE VIIa, where a peak displayed at the region of $1024.27 \mathrm{~cm}^{-1}$ corresponded to the methoxy group.

Further confirmation of chemical composition of LCPUE produced was carried out by means of Nuclear Magnetic Resonance spectroscopy (NMR). ${ }^{1} \mathrm{H}-\mathrm{NMR}$ spectrum of LCPUE VIIa was illustrated in Figure.2. A singlet peak centered at $8.76 \mathrm{ppm}$ was assigned to NHCOO- and this proved the formation of urethane linkage. The appearance of multiplet peaks at 7.53-6.99 ppm and singlet peak at $3.87 \mathrm{ppm}$ was attributed to the aromatic protons and the protons in methoxy group, respectively. Aliphatic chain of polyol (PEG 1000) was detected in the region of 1.23-1.64 ppm.

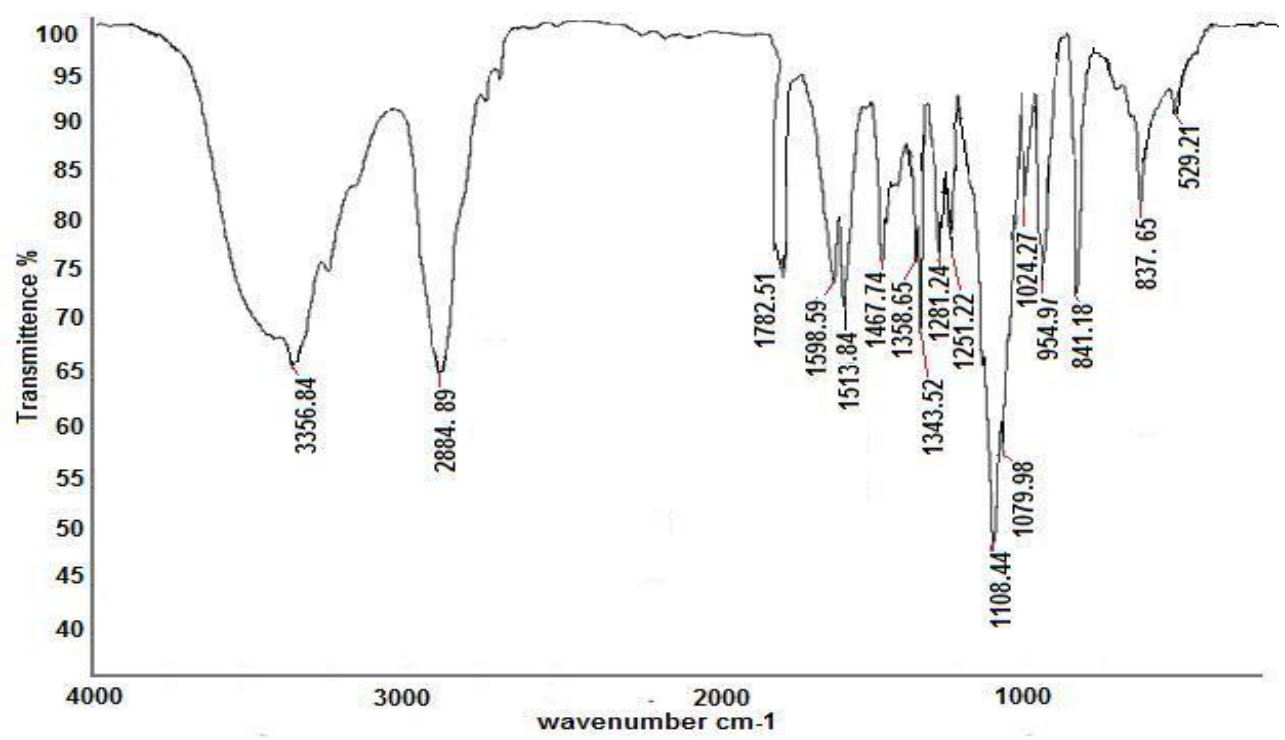

Figure 1. FTIR spectrum of LCPUE VIIa 


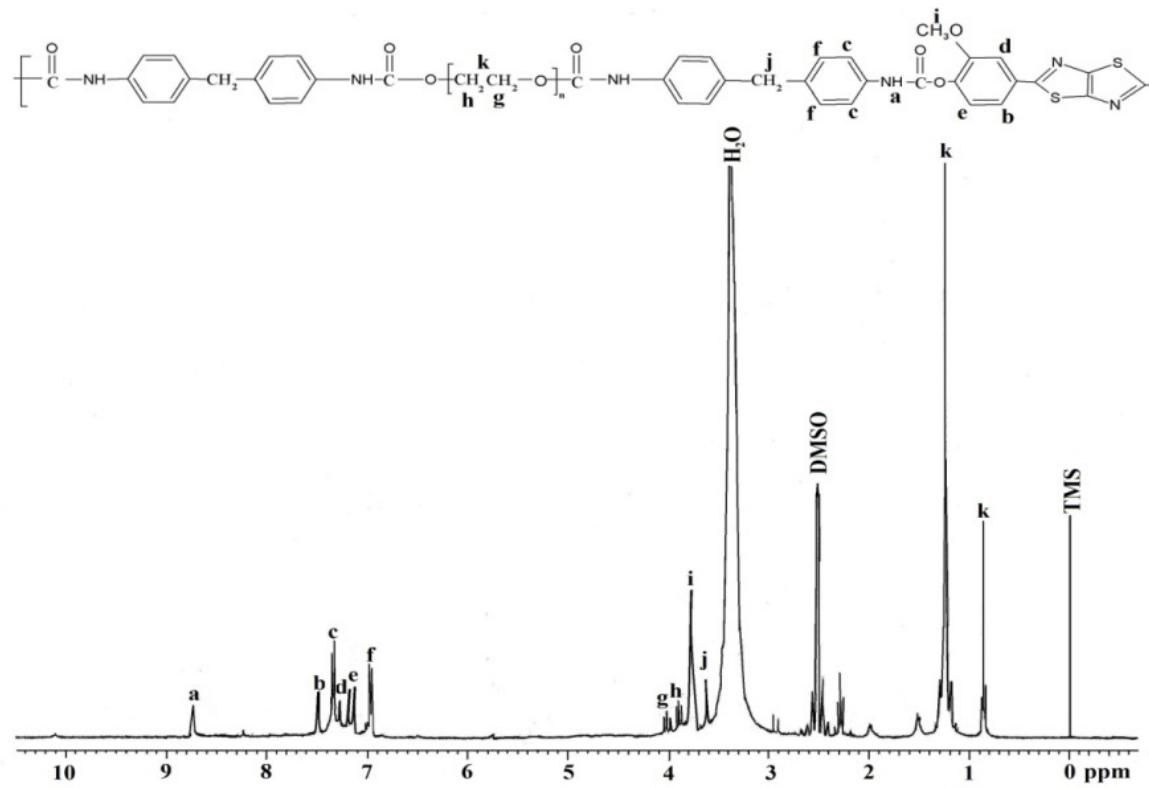

Figure 2. ${ }^{1} \mathrm{H}-\mathrm{NMR}$ spectrum of LCPUE VIIa
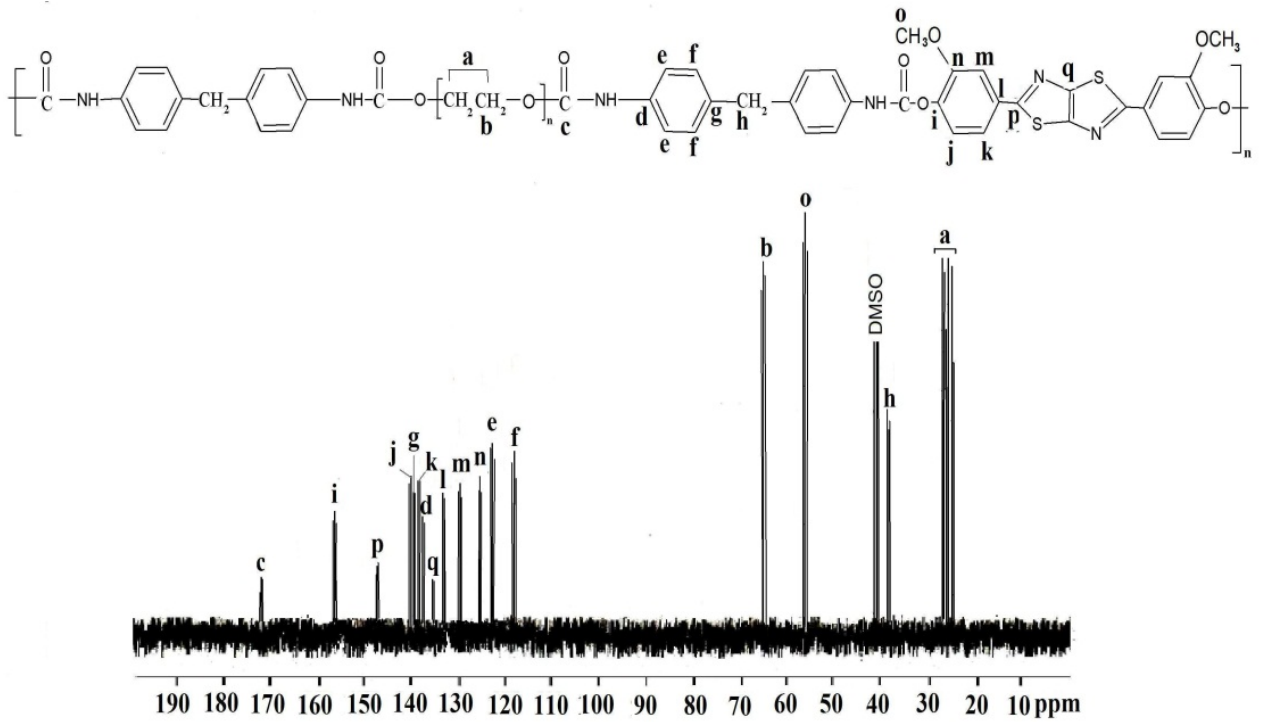

Figure 3. ${ }^{13} \mathrm{C}-\mathrm{NMR}$ spectra of LCPUE VIIa

Other than FT-IR and ${ }^{1} \mathrm{H}-\mathrm{NMR}$ analysis, ${ }^{13} \mathrm{C}-\mathrm{NMR}$ was performed in order to clarify the structure of LCPUE prepared. ${ }^{13} \mathrm{C}-\mathrm{NMR}$ spectra portrayed in Figure.3 which represents LCPUE VIIa shows that the formation of urethane linkage (NHCOO) was determined by the 
appearance of the peak at $173.4 \mathrm{ppm}$. The methylene group presence in the soft segment of PEG can be seen as a sharp and intense peak at 25-29 ppm. More peaks can be observed at 117.8 to $158.7 \mathrm{ppm}$ and $56.2 \mathrm{ppm}$ where they were assigned to the aromatic carbons and the carbon in methoxy group respectively. Significant peaks in all characterization analysis (FT-IR, ${ }^{1} \mathrm{H}-\mathrm{NMR}$ and ${ }^{13} \mathrm{C}-\mathrm{NMR}$ ) were consistent and adequately provide the evidences to support the fact that the reaction of all materials took place and LCPUE was successfully prepared.

\subsection{Thermal and liquid crystalline behavior of polymers}

The DSC analysis was conducted at a heating rate of $10^{\circ} \mathrm{C}$ to understand phase separation behavior of all synthesized LPCUE where the transition occurs, observed under polarizing optical microscope (POM) equipped with heating stage and the results obtained from both measurements were listed in Table 1. Based on the DSC thermograms, upon heating, one step transition and two endothermic peaks were detected where each of them indicates glass transition $\left(\mathrm{T}_{\mathrm{g}}\right)$, melting endotherm, $\left(\mathrm{T}_{\mathrm{m}}\right)$ and isotropic endotherm $\left(\mathrm{T}_{\mathrm{i}}\right)$ respectively, which is also the evidence of the existence of mesophase. LCPUE derived from 2,5-bis(4-hydroxy-3methoxyphenyl) thiazolo [5,4d] thiazole have transition temperatures lower than those derived from 2,5-bis(4-hydroxyphenyl)thiazolo-[5,4d]thiazole. Methoxy group, which acts as a substituent attached to the phenyl ring has the capability to lower the melting and isotropization temperature and caused thermal suppression of the molecule to occur (AlDujaili et.al., 2001). The fact was supported by the results illustrated in Fig. 4 where it depicts the DSC thermograms of LCPUE. LCPUE VIIa displayed melting point $\left(\mathrm{T}_{\mathrm{m}}\right)$ at $164^{\circ} \mathrm{C}$ and isotropization temperature $\left(\mathrm{T}_{\mathrm{i}}\right)$ at $187^{\circ} \mathrm{C}$ whereas for LCPUE VIa, $\mathrm{T}_{\mathrm{m}}$ was detected at $176^{\circ} \mathrm{C}$ and $\mathrm{T}_{\mathrm{i}}$ at $205^{\circ} \mathrm{C}$. The substituent could also act to reduce the coplanarity of adjacent mesogenic groups and increase the diameter or decrease the axial ratio of the mesogens [Li and Chang, 1991]. Due to the higher range between $T_{m}$ and $T_{i}$ of LCPUE VIa, the thermal properties of this polymer are higher and more stable compared to LCPUE VIIa. The types of diisocyanates also contribute to the thermal behavior of LCPUE, where MDI based PU was known for having better order of the rigid chain that approaches the decomposition temperature, giving high melting point to the polymer produced (Jieh \& Chou, 1996). As for glass transition, it involves mobility of the chain segments and the Tg will be affected by the mobility restriction on the chain segments, (Suresh et.al., 2008) it therefore explains the varying pattern of the $\mathrm{T}_{\mathrm{g}}$ values displayed in Table 1 . The decreasing values of $\mathrm{T}_{\mathrm{g}}$ can be observed as the length of soft segments increases, indicating that the long chain of polyol gave great flexibility characteristics towards the polymer chains where less mobility restrictions occurred and hence resulting in the lower $\mathrm{T}_{\mathrm{g}}$ values.

POM was utilized to investigate the type of mesophase by displaying the phase transition that occurred, subsequently providing the polarizing optical microphotographs of the target compounds. The morphology observed on heating and transition temperatures obtained were given in Figures 5 and 6 and the results were summarized in Table 1. It was revealed that all LCPUE showed mesophases upon melting temperature where the thread texture of the nematic phases can be seen. From the photographs taken by POM, the crystal to mesophase transition occurred at temperature ranging from 129 to $181^{\circ} \mathrm{C}$. The samples were 
further heated after the crystal-nematic transition temperature, and resulted in the disappearing of the texture when reaching the isotropization stage. There were no traces of mesophase transition during the cooling process from POM indicating all samples possessed thermotropic type of liquid crystal. Phase transition temperatures observed through POM were found to be consistent with the corresponding DSC thermograms.

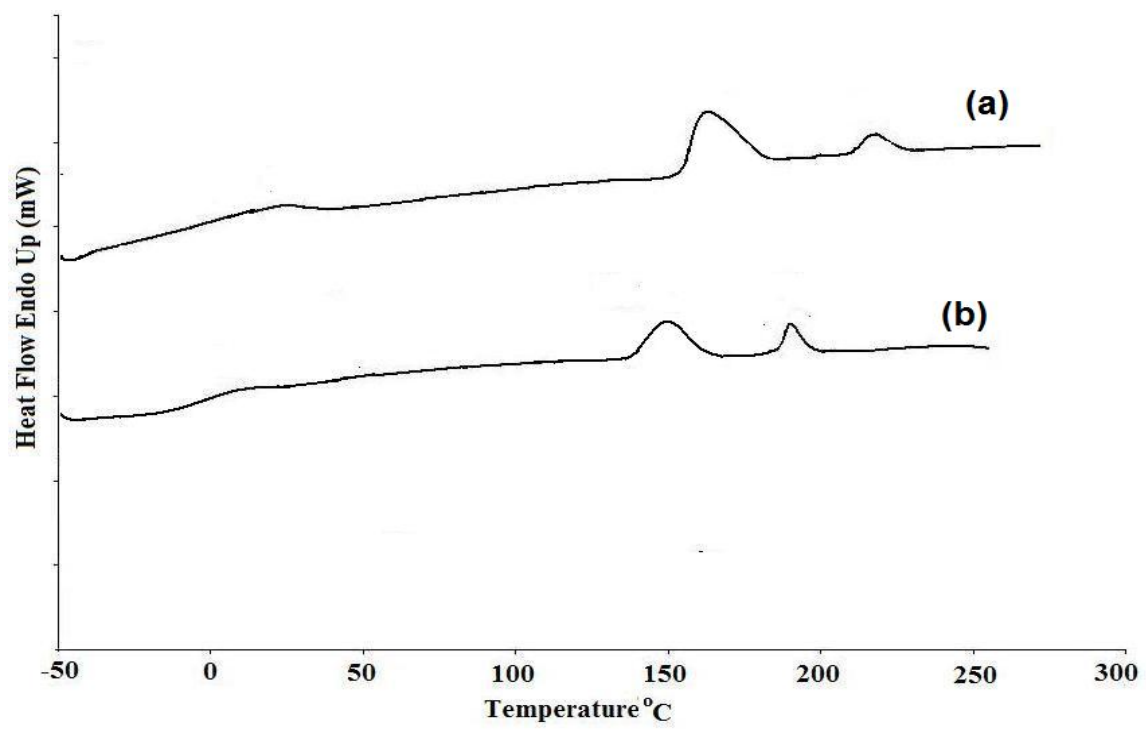

Figure 4. DSC traces of (a) LCPUE VIa (b) LCPUE VIIa

\begin{tabular}{|c|c|c|c|c|c|c|c|c|}
\hline \multirow[b]{2}{*}{ SAMPLE } & \multirow[b]{2}{*}{$\begin{array}{c}\text { PEG MOLECULAR } \\
\text { WEIGHT }\end{array}$} & \multirow[b]{2}{*}{$\begin{array}{l}\text { Yield } \\
(\%)\end{array}$} & \multirow[b]{2}{*}{$\begin{array}{c}\text { Viscosity } \\
\text { cP }\end{array}$} & \multicolumn{3}{|c|}{ DSC } & \multicolumn{2}{|c|}{ POM } \\
\hline & & & & $\begin{array}{c}\mathrm{T}_{\mathrm{g}} \\
\left({ }^{\circ} \mathrm{C}\right)\end{array}$ & $\begin{array}{c}\mathrm{T}_{\mathrm{m}} \\
\left({ }^{\circ} \mathrm{C}\right)\end{array}$ & $\begin{array}{c}\mathrm{T}_{\mathrm{i}} \\
\left({ }^{\circ} \mathrm{C}\right)\end{array}$ & $\begin{array}{c}\mathrm{T}_{\mathrm{m}} \\
\left({ }^{\circ} \mathrm{C}\right)\end{array}$ & $\begin{array}{c}\mathrm{T}_{\mathrm{i}} \\
\left({ }^{\circ} \mathrm{C}\right)\end{array}$ \\
\hline LCPUE VIa & 1000 & 85 & 11108 & 25.1 & 176 & 205 & 181 & 200 \\
\hline LCPUE VIb & 2000 & 83 & 26456 & 22.5 & 153 & 174 & 162 & 180 \\
\hline LCPUE VIC & 3000 & 77 & 40692 & 19.1 & 139 & 156 & 133 & 161 \\
\hline LCPUE VIIa & 1000 & 76 & 10744 & 15.2 & 164 & 187 & 170 & 193 \\
\hline LCPUE VIIb & 2000 & 80 & 22453 & 11.8 & 143 & 163 & 148 & 170 \\
\hline LCPUE VIIC & 3000 & 87 & 39981 & 10.4 & 125 & 142 & 129 & 149 \\
\hline
\end{tabular}

Table 1. Thermal properties of LCPUE VI (a-c) and LCPUE VII (a-c) by DSC and POM 


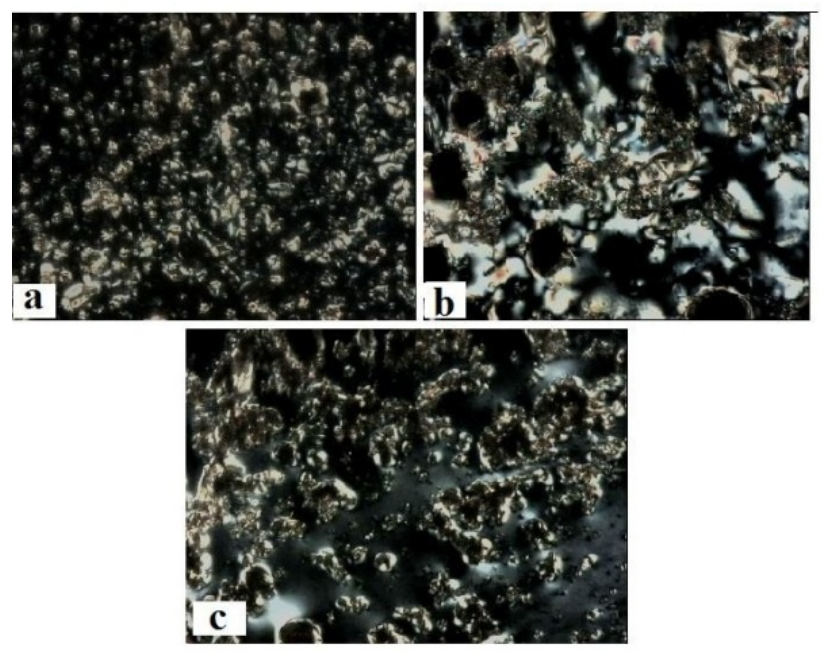

Figure 5. Polarized optical images of (a) LCPUE VIa $\left(181^{\circ} \mathrm{C}\right)$, (b) LCPUE VIb $\left(162^{\circ} \mathrm{C}\right)$ and (c) LCPUE VIc $\left(133^{\circ} \mathrm{C}\right)$
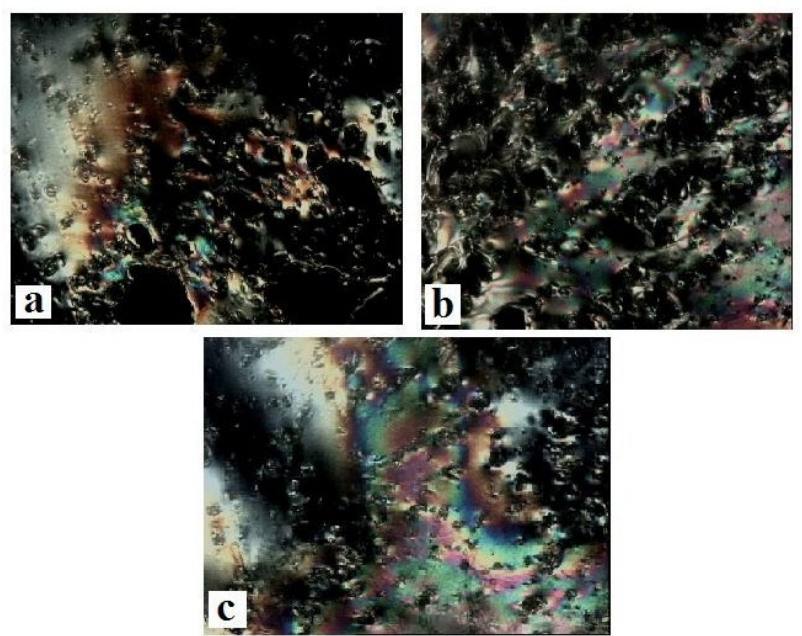

Figure 6. Polarized optical images of (a) LCPUE VIIa $\left(170^{\circ} \mathrm{C}\right)$, (b) LCPUE VIIb $\left(148^{\circ} \mathrm{C}\right)$ and (c) LCPUE VIIc $\left(129^{\circ} \mathrm{C}\right)$

X-ray diffraction analysis of LCPUE was conducted at room temperature to obtain information on both the mesophase structure and crystallinity of LCPUE. The measurements exhibited several peaks in the range of $2 \theta=15-25^{\circ}$ as observed in Figure 7 
and this indicated semi crystalline character possessed by LCPUE. The results obtained in above range also provide details related to the d-spacing of 3.56 and $4.92 \AA$, thus supporting the characteristic of nematic liquid crystalline phase (Jeh \& The, 1994) as displayed through POM.

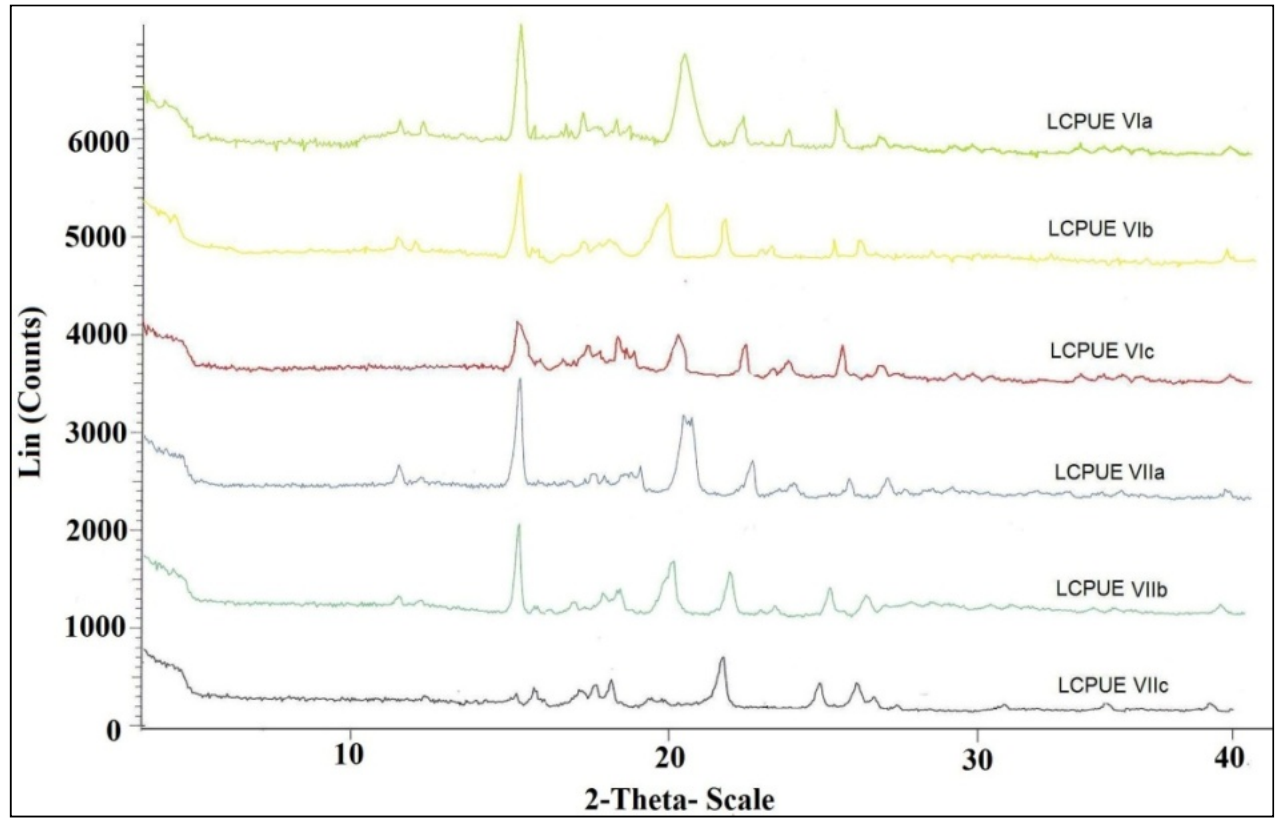

Figure 7. X-ray diffraction scales of LCPUE VI (a-c) and LCPUE VII (a-c)

Thermal stability of prepared LCPUE was investigated by thermogravimetric analysis (TGA). Incorporation of liquid crystalline properties into the polymer structure would enhance the thermal properties (Jahromi et.al., 1994) and the theory has proved to be applicable from the results obtained. This may be partly due to favorable interactions between hard domain interface and the liquid crystalline phase. All synthesized LCPUE possessed good thermal stabilities, however, PU elastomers eventually undergo thermal degradation when exposed to high temperatures. Degradation process occurred in two step pattern where the initial degradation occurs in the hard segment involving the urethane linkages, while the second stage indicated the degradation of soft segments. TGA curves in Figure 8 demonstrated the thermal degradation of all LCPUE prepared where $10 \%$ weight loss of LCPUE occurred at about $315-341^{\circ} \mathrm{C}$ and the maximum degradation temperature was in the range of $430-470^{\circ} \mathrm{C}$, signifying a high thermal stability property. Furthermore, it can be observed that LCPUE VIIc demonstrated the lowest degradation temperature among the others and this proved that the length of polyethylene glycol (soft segment) influenced the thermal stability of LCPUE where the order of LCPUE due to their thermal stability can be arranged as LCPUE VIa $>$ VIIa $>$ VIb $>$ VIIb $>$ VIc $>$ VIIc. 


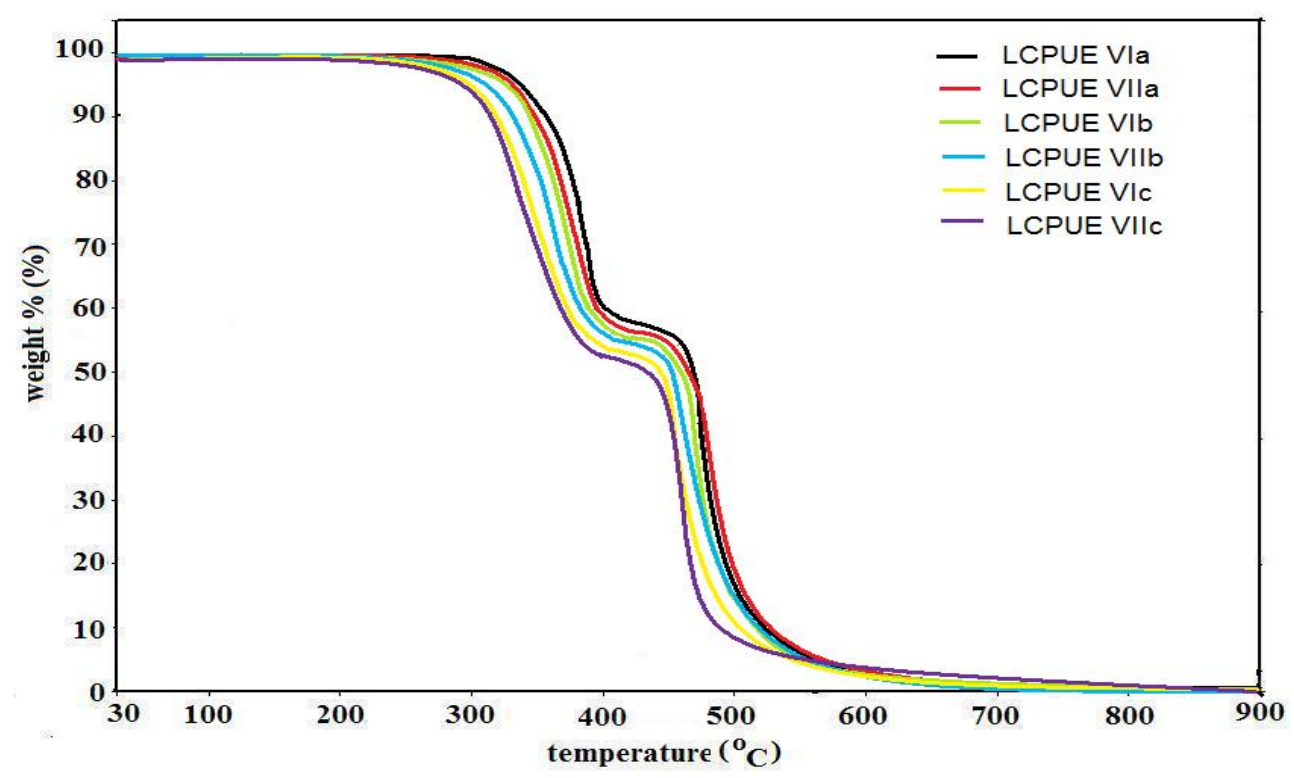

Figure 8. TGA curve of LCPUE VI (a-c) and LCPUE VII (a-c)

\subsection{Tensile properties}

Table 2 demonstrates tensile properties of the synthesized LCPUE. As seen, all of the polymers possessed good elastic properties with high elongation at break. Due to the data listed, the higher the molecular weight of the soft segments, the greater the elongation at break, but decrease of tensile strength and tensile modulus can be observed. When the molecular weight of polyol increased, the number of urethane groups in the polyol chain was reduced at the same time, and hence the number of rigid segments is lower, consequently, the possible number of intermolecular hydrogen bonds goes down in which $-\mathrm{NH}$ and $\mathrm{C}=\mathrm{O}$ groups are active (Kro \& Pitera, 2008). However, the presence of enhanced rigid and high aspect ratio mesogenic unit as part of hard segment in the synthesized LCPUE, is able to give both high strength and good elastic properties to LCPUE even with long soft segments, which

\begin{tabular}{cccc}
\hline Sample & $\begin{array}{c}\text { Tensile modulus } \\
(\mathrm{Mpa})\end{array}$ & $\begin{array}{c}\text { Tensile strength } \\
(\mathrm{Mpa})\end{array}$ & $\begin{array}{c}\text { Elongation at break } \\
(\%)\end{array}$ \\
\hline LCPUE VIa & 17.1 & 28.2 & 290 \\
LCPUE VIb & 13.4 & 24.1 & 450 \\
LCPUE VIc & 11.5 & 19.9 & 570 \\
LCPUE VIIa & 17.5 & 28.3 & 330 \\
LCPUE VIIb & 13.7 & 24.3 & 460 \\
LCPUE VIIc & 11.2 & 19.8 & 560 \\
\hline
\end{tabular}

Table 2. Mechanical properties of LCPUE VI (a-c) and LCPUE VII (a-c) 
is unusual in conventional PUE (Jeong et.al., 2000). Better phase separation will lead to good mechanical properties; hence the introduction of the mesogens unit as chain extender into LCPUE can be said to easily induce the matter (phase separation) to occur.

\section{Author details}

Mohammed Ahmed Issam and Hamidi Mohamed Rashidah

University Sains Malaysia, Malaysia

\section{Acknowledgement}

The author would like to thank University Sains Malaysia for short term grant no.304.PTEKIND.6311031 and the fellowship scheme for funding the research.

\section{References}

Abe, A. \& Ballauf, M. (1991). Liquid crystallinity in Polymers. John Wiley \& Sons Inc, New York, USA

Al-Dujaili, A.H.; Atto, A.T. \& Al-Kurde, A.M. (2001). Synthesis and Liquid Crystalline Properties of Models and Polymers containing Thiazolo[5,4-d]thiazole and Siloxane Flexible Spacers. European Polymer Journal Vol.37, pp. 927-932

Bagheri, M. \& Pourmoazzen, Z. (2008). Synthesis and Properties of New Liquid Crystalline Polyurethanes containing Mesogenic Side Chain Reactive \& functional. Polymers, Vol.68, pp. 507-518

Barikani, M.; Honarkar, H. \& Barikani, M. (2009). Synthesis and Characterization of Polyurethane Elastomers based on Chitosan and Poly(e-caprolactone). Journal of Applied Polymer Science, Vol.112, pp. 3157-3165

Doldeny, J.D. \& Alder, P.T. (1998). The Mesogenic Index: An Empirical Method for Predicting Polymeric Liquid Crystallinity. High Performance Polymers, Vol.10, pp. 249-272

Issam, A.M. (2007). Synthesis of Novel Y-Type Polyurethane containing Azomethine Moiety, as Non-linear Optical Chromophore and Their Properties. European Polymer Journal, Vol.43, pp. 214-219.

Jahromi, S.; Lub, J. \& Mol, G.N. (1994). Synthesis and Photoinitiated Polymerization of Liquid Crystalline Diepoxides. Polymer, Vol. 35, No.3, pp. 622-629

Jeh, C.T. \& Teh, C.C. (1994). Study on Thermotropic Liquid Crystalline Polymers -I. Synthesis and Properties of Poly(azomethine-urethane)s. European Polymer Journal, Vol.30, pp. 1059-1064

Jeong, H.M.; Kim, B.K. \& Choi, Y.J. (2000). Synthesis and Properties of Thermotropic Liquid Crystalline Polyurethane Elastomers. Polymer,Vol.41, pp. 1849-185

Jia, X.; He, X.D. \& Yu, X.H. (1996). Synthesis and Properties of Main-Chain liquid Crystalline Polyurethane Elastomers with Azoxybenzene. Journal of Applied Polymer Science, Vol.62, pp. 465-47

Jieh, S.S. \& Chou, C.T. (1996). Studies on Thermotropic Liquid Crystalline Polyurethanes.III.Synthesis and properties of polyurethane elastomers by using various 
mesogenic units as chain extender. Journal of polymer science part A: Polymer chemistry, Vol.34, pp. 771-779

Knight, D.P. \& Vollrath, F. (2002). Biological Liquid Crystal elastomers. Philosophical Transactional Royal Society London. B, Vol.357, pp. 155-163

Knighton, R.C.; Hallett, A.J.; Kariuki, B.M. \& Pope, S.J.A. (2010). A One-step Synthesis towards New Ligands based on Aryl-functionalized Thiazolo[5,4-d]thiazole Chromophores. Tetrahedron Letters, Vol.51, pp. 5419-5422

Kro, P. \& Pitera, B.P. (2008). Mechanical Properties of Crosslinked Polyurethane Elastomers Based on Well-Defined Prepolymers. Journal of Applied Polymer Science, Vol.107, No.3, pp. 1439-1448

Lee, D.J.; You, S.H. \& Kim, H.D. (1999). Synthesis and properties of thermotropic liquid crystalline polyurethane elastomers (II): Effect of Structure of Chain Extender Containing Imide Unit. Korea Polymer Journal, Vol.7, No.6, pp. 356-363

Lin, C.K.; Kuo, J.F. \& Chen, C.Y. (2001). Synthesis and Properties of Novel Polyurethanes containing the Mesogenic Moiety of a-Methylstilbene Derivatives. European Polymer Journal, Vol.37, pp. 303-313

Li, C.H.; \& Chang, T.C. (1991). Thermotropic Liquid Crystalline Polymer:III: Synthesis and Properties of Poly(amide-azomethine-ester). Journal of Polymer Science Part A: Polymer Chemistry, Vol.29(3), pp. 361-367

Osaka, I.; Sauvé, G.; Zhang, R.; Kowalewski, T. \& McCullough, R.D. (2007). Novel Thiophene-Thiazolothiazole Copolymers for Organic Field-Effect Transistors. Advance Material, Vol.19, pp. 4160-4165

Pacheco, M.F.M.; Bianchi, O.; Fiorio, R.; Zattera, A.J.; Giovanel, M.Z.M. \& Crespo, J.S. (2009). Thermal, Chemical, and Morphological Characterization of Microcellular Polyurethane Elastomers. Journal of Elastomers and Plastics, Vol.41, pp. 323

Rowan, S.J. \& Mather, P.T. (2008). Supramolecular Interactions in the Formation of Thermotropic Liquid Crystalline Polymers. Structure and bonding, Vol.128, pp. 119-149

Suresh, K.I.; Tamboli, J.R.; Rao, B.S.; Verma, S. \& Unnikrishnan G. (2008). Effect of Core Group Substituents on the Monomer Mesophase, Photocuring, and Film Viscoelastic Properties of Mesogenic Diacrylates. Polymers for Advanced Technologies, Vol.19, pp.1323-1333

Wright, P. \& Cumming, A.P.C. (1969). Solid Polyurethane elastomers. Mclaren and Sons, London

Yeganeh, H. \& Mehdizadeh, M.R. (2004). Synthesis and Properties of Isocyanate Curable Millable Polyurethane Elastomers Based on Castor Oil as a Renewable Resource Polyol. European Polymer Journal, Vol.40, pp. 1233-1238

Yeganeh, H.; Talemi, P.H. \& Jamshidi, S. (2007). Novel Method for Preparation of Polyurethane Elastomers with Improved Thermal Stability and Electrical Insulating Properties. Journal of Applied Polymer Science, Vol.103, pp. 1776-1785

Zhang, C.; He, Z .; Wang, J.; Wang, Y. \& Ye, S. (2008). Study of Mesogenic Properties and Molecular Conformation from a Heterogeneous Tetramer with a Triphenylene Centre Core and Three Cyanobiphenyl Tails. Journal of Molecular Liquids, Vol. 138, pp. 93-99

Zhang, H.; Chen, Y.; Zhang, Y.; Sun, X.; Ye, H. \& Li, W. (2008). Synthesis and Characterization of Polyurethane Elastomers. Journal of Elastomers and Plastics, Vol.40, No.2, pp. 161-177 